\title{
Road friendliness optimization of heavy vehicle suspension based on particle swarm algorithm
}

\author{
Lufeng Wang ${ }^{1,2, a}$, Zheng $\mathrm{Lv}^{2, \mathrm{~b}}$ and Qi Li $\mathrm{i}^{2, \mathrm{c}}$ \\ ${ }^{1}$ Neimenggu Jiaotong Zhiye Jishu Xueyuan, Chifeng 024005, China; \\ ${ }^{2}$ School of Aeronautic Science and Engineering, Beihang University, Beijing 100191, China. \\ a_f_wang@126.com, blvbaolvzheng@126.com, 'Iq1213@163.com
}

Keywords: particle swarm optimization, heavy vehicle suspension, optimization design, time domain analysis, frequency domain analysis.

\begin{abstract}
In this paper, an optimization design method based on the Particle Swarm Optimization (PSO) technique is proposed for the enhancement of the passive suspension system performance of heavy vehicles. The optimization problem based on a quarter-vehicle model aims to minimize the dynamic tyre load subject to constraints on the natural frequency of the unsprung mass. PSO is applied to solve the optimization problem for obtaining optimum parameters, such as the suspension damping coefficient, spring and tyre stiffness. In order to assess the obtained design parameters, the suspension performance is estimated in time and frequency domains for different road excitations. The results show that the proposed method can obtain the optimum designed parameters quickly compared with the previous designed parameters using the genetic algorithm for the same passive suspension system, which will provide important parameters for the subsequent revised design. This paper provides a feasible way for the optimization design of a new type of suspension system.
\end{abstract}

\section{Introduction}

Road friendliness of a vehicle is significantly influenced by the road surface roughness, the speed of the vehicle and its suspension system. Due to the difficulty of improving the road surface roughness, it becomes a prevailing philosophy for vehicle designer to design a good suspension system with optimum vibration performance in the vehicle industry.

Nowadays, the most popular suspension system used in heavy vehicles is still passive suspension. Two principal parameters for design of this suspension are spring stiffness and damper coefficient $[1,2]$. The optimal parameters should overcome the conflicting requirements that the hard damper gives better stability but reduced ride comfort whereas the soft one offers the contrary effect [3-6].

As the suspension design criterion, ride quality mainly stems from taking the human ride comfort perspective. From a cost-effectiveness of tyre-terrain systems perspective, on the other hand, the dynamic tyre load applied on the pavement is also important performance requirements especially for the heavy vehicles [7-10].It has been recognized from several years ago that pavement damages are related to the dynamic tyre load through a fourth power law [7]. That is to say, a $10 \%$ increase in the dynamic tyre load will cause an approximately 50\% increase in pavement damage [11]. Considering the massive maintenance cost of road pavement, an approach for road friendliness optimization of heavy vehicle suspension using dynamic tyre load as a design criterion has been developed.

Conventionally, the objective function used in the suspension system design is usually highly nonlinear and may possess multiple extreme [12].The optimal solution obtained by using classical optimization algorithms which require the gradient to be computed during the iterative search process may often be trapped in a local optimum [13]. Under such circumstance, researchers applied genetic algorithm to vehicle suspension design [11, 14-16]. However, further studies show that a lot of parameters for the optimal solution must be properly settled on.

Particle swarm algorithm developed by Kennedy and Eberhart [17, 18] is randomized search techniques guided by the principles of the movement of organisms in a bird flock or fish school. The main advantages of particle swarm algorithm are simplicity, robustness and good convergence. The 
studies [19-21] find that the parameters identification in particle swarm algorithm is more robustly controlled and more simply selected than GA. To take advantage of particle swarm algorithms as well as to consider more realistic road surfaces, we implement particle swarm algorithms in this paper to solve the optimum vehicle suspension design problem.

\section{Quarter car model and road excitation}

Considering the two degree of freedom system which simulates the quarter car suspension illustrated in Fig. 1, the dynamic equilibrium equations of the model is given as

$$
\begin{aligned}
& m_{b} \ddot{x}_{b}+k_{s}\left(x_{b}-x_{w}\right)+c_{s}\left(\dot{x}_{b}-\dot{x}_{w}\right)=0 \\
& m_{w} \ddot{x}_{w}-k_{s}\left(x_{b}-x_{w}\right)-c_{s}\left(\dot{x}_{b}-\dot{x}_{w}\right)+k_{t}\left(x_{w}-x_{r}\right)=0
\end{aligned}
$$

where $m_{b}$ and $m_{w}$ are the chassis body mass and the wheel mass, respectively. $k_{s}$ and $c_{s}$ are the suspension spring stiffness and the damping coefficient, respectively. $k_{t}$ is the tyre spring stiffness while its damping was neglected. $x_{b}$ and $x_{w}$ are the vertical displacements of $m_{b}$ and $m_{w}$, respectively. $x_{r}$ is the road disturbance.

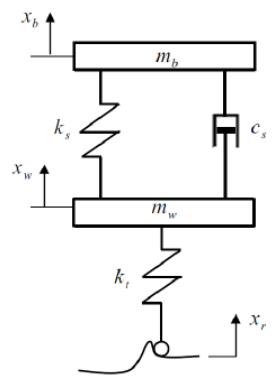

Fig. 1 Quarter vehicle suspension model

The undesirable vehicle body vibration is mostly the result of the road surface irregularities, including the discrete or periodic irregularities and the random irregularities. A typical road surface which reflects the transient response characteristic is given as

$$
x_{r}=\left\{\begin{array}{l}
a\left\{1-\cos \left(\omega_{r}(t-0.5)\right)\right\}, 0.5 \leq t \leq 0.5+\frac{d}{V} \\
0, \text { otherwise }
\end{array}\right.
$$

where $a=0.035 \mathrm{~m}$ is the half of the bump amplitude, $d=0.8 \mathrm{~m}$ is the width of the bump, and $V=3.08 \mathrm{~km} / \mathrm{s}$ is the vehicle speed.

The road random surface irregularities caused by environmental action are in general treated as stationary random processes described by power spectral densities. A stationary Gaussian random process is used in frequency analysis to reflect the response characteristic which defined in[23] as

$$
S_{x}(f)=G V^{n-1} / f^{n}
$$

where $G=5 \times 10^{-6}$ is the road surface roughness coefficient, $f=0.25 \mathrm{~Hz}$ is the road surface frequency, $n=2.5$ is the road roughness exponent, and $V=20 \mathrm{~m} / \mathrm{s}$ is the vehicle speed.

\section{Road friendliness optimization based on particle swarm algorithm}

A good suspension system requires the matching design of the spring stiffness and the damping coefficient. The main aim of solving road friendliness optimization problem of heavy vehicle is to minimize the dynamic tire load (DTL), which significantly influences the ride comfort, vehicle stability and the life time of the pavement. To this end, it is required to evaluate the optimum values of the suspension parameters to minimize the maximum DTL. Based on the discussions above, the nonlinear optimization model for vehicle suspension is defined as: 


$$
\begin{aligned}
& \text { find } X=\left(k_{t}, k_{s}, c_{s}\right) \\
& \min f(X)=\max |D T L| \\
& \text { s.t. } \begin{cases}1 e+5 \leq k_{t} \leq 3 e+6, & 1.5 e+6 \leq k_{s} \leq 2 e+6 \\
1 e+4 \leq c_{s} \leq 3 e+5, & 9.8 \leq \omega \leq 10.2\end{cases}
\end{aligned}
$$

where $\omega$ is the natural frequency of the unsprung mass. The range of the design variables $k_{t}, k_{s}, c_{s}$ and $\omega$ are referred to the literatures [11, 22].

The main steps involved in the particle swarm algorithm are summarized: 1) initialization of particles; 2) evaluation of the objective function; 3) update the position and velocity of each particle. Thus, the optimization problem in Eq.(4) can be solved according to the flowchart of optimization procedure shown in Fig. 2, where the position and velocity of each particle are updated as follows

$$
\begin{aligned}
& y_{p}^{(j+1)}=y_{p}^{(j)}+v_{p}^{(j+1)} \\
& v_{p}^{(j+1)}=W \times v_{p}^{(j)}+c_{1} \times \operatorname{rand}_{1} \times\left[b_{p}^{(j)}-y_{p}^{(j)}\right]+c_{2} \times \operatorname{rand}_{2} \times\left[g_{p}^{(j)}-y_{p}^{(j)}\right]
\end{aligned}
$$

where $y_{p}^{(j)}, v_{p}^{(j)}$ are the position and velocity of the particle at the generation $j$. W is the inertia weight, $C_{1}, c_{2}$ are acceleration coefficients, rand $_{1}$, rand $_{2}$ are random numbers which belong to $[0,1]$.

$$
\text { Calculate the fitness function of particles }
$$

Fig. 2 Flowchart of optimization procedure

The optimum solutions to Eq.(4) for various numbers of iterations $N_{i}$ and particles $N_{p}$ are listed in Table 1. We can see that the optimum solution strongly relies on the numbers of iterations and particles. The optimum solution is stable for the case of $N_{i}=2000$ with $N_{p} \geq 70$, which demonstrates the convergence of the objective function of road friendliness optimization problem.

Table 1 Minimum values for the maximum dynamic tyre load $(\mathrm{kN})$

\begin{tabular}{cccccccc}
\hline \multirow{2}{*}{$N_{p}$} & \multicolumn{7}{c}{$N_{i}$} \\
\cline { 2 - 8 } & 30 & 70 & 100 & 300 & 700 & 1000 & 2000 \\
\hline 30 & 14.45 & 13.50 & 13.22 & 12.34 & 12.55 & 12.58 & 12.49 \\
70 & 13.22 & 12.82 & 12.77 & 12.74 & 12.16 & 12.17 & 12.13 \\
100 & 13.20 & 13.90 & 13.29 & 12.77 & 12.22 & 12.42 & 12.13 \\
300 & 13.30 & 13.14 & 13.14 & 12.35 & 12.14 & 12.13 & 12.13 \\
\hline
\end{tabular}

\section{Results and discussions}

Dynamic tyre load, body acceleration (BA) and suspension working space (SWS) are the major system behavior criteria for the design of vehicle suspension that related to ride comfort and vehicle stability. To demonstrate the effectiveness of the proposed method, performance comparison of suspension obtained by PSO and GA is done. The quarter vehicle optimum suspension parameters are listed in Table 2, where the optimum parameters are obtained by PSO for the case of $\mathrm{Ni}=2000$ with $\mathrm{Np}=70$, and the parameters obtained using GA for the same passive suspension system of heavy vehicle are referred to the literature [11]. 
Table 2 Quarter vehicle optimum suspension parameters

\begin{tabular}{cccc}
\hline Parameter & Symbol & Value GA & Value PSO \\
\hline Vehicle mass (kg) & $m_{b}$ & 4,450 & 4,450 \\
Wheel mass (kg) & $m_{w}$ & 550 & 550 \\
Suspension stiffness (N/m) & $k_{s}$ & 622,180 & 123,807 \\
Damping coefficient (N.s/m) & $c_{s}$ & 26,582 & 21,053 \\
Tyre stiffness (N/m) & $k_{t}$ & $1,705,449$ & $1,997,216$ \\
\hline
\end{tabular}

\subsection{Time domain analysis}

Fig.3 shows the time history of the vehicle suspension systems behavior for the bump road profile given in Eq.(2). It is clearly seen that the suspension obtained using the PSO has the lowest peaks for the BA and DTL. Compared with the suspension obtained using GA, the maximum peak-to peak (PTP) values of BA and DTL can be reduced by $57.4 \%$ and $58.3 \%$, respectively. Furthermore, although the PTP value of the SWS using PSO is higher than that using GA, the number of vibrating cycles is reduced by half, which is useful for the extension of the life time of the suspension elements. Obviously, the suspension obtained using PSO can waste the energy due to bump excitation effectively, cut down the settling time, and improve both the ride comfort and vehicle stability.

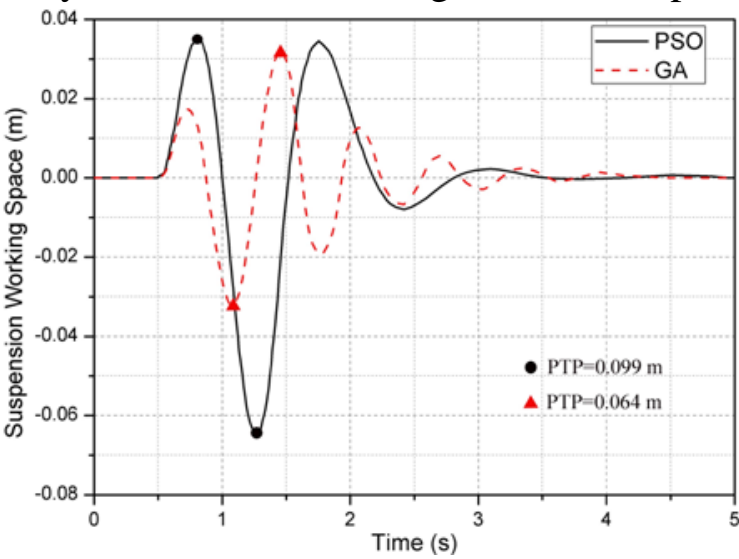

(a) SWS

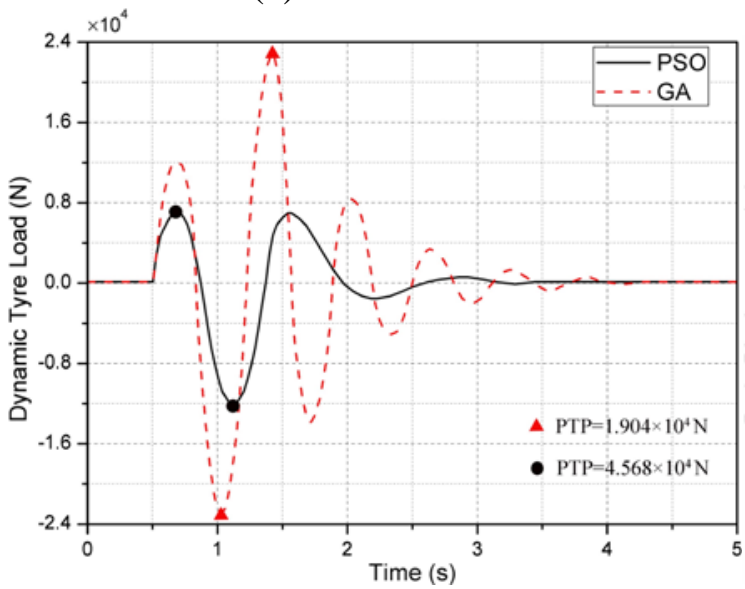

(c) DTL

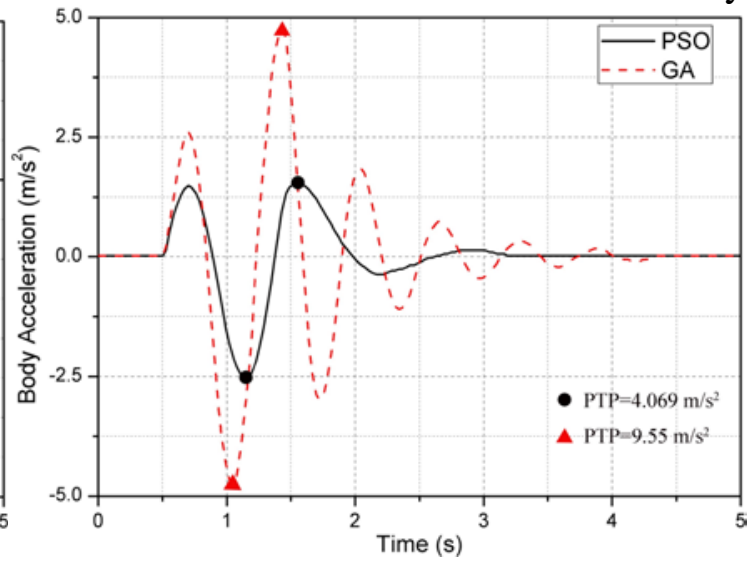

(b) BA

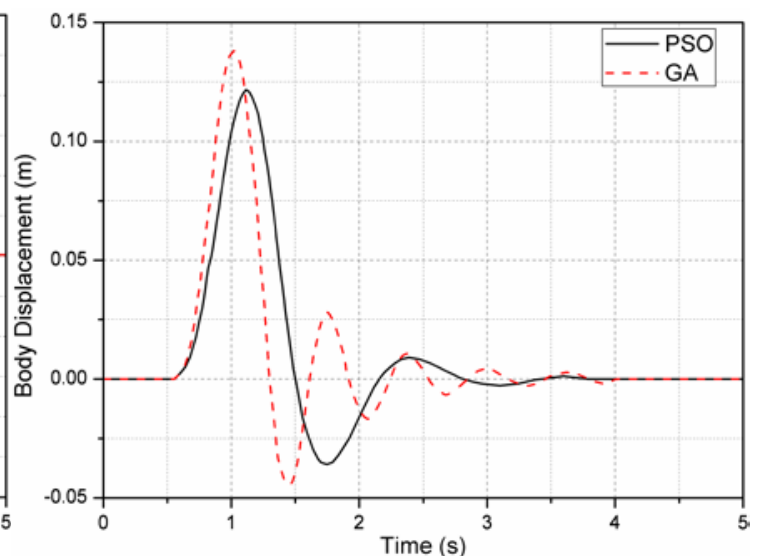

(d) Body Displacement

Fig. 3 The time history of system response under bump road excitation

\subsection{Frequency domain analysis}

Fig. 4 shows the power spectral density of the vehicle suspension systems behavior over the frequency range $0.25-20 \mathrm{~Hz}$. In frequency domain analysis, we are interest in the root-mean-square (RMS) values of SWS, BA and DTL, instead of their PTP values. From Fig. 5, it can be seen that the suspension obtained using the PSO has the lowest levels of RMS values for the BA and DTL. The maximum RMS values of BA and DTL are, respectively, reduced by $40.8 \%$ and $26.1 \%$ compared with the suspension obtained using GA. The results demonstrate once more the suspension obtained using the PSO can offer superior performance to meet the requirement of the ride comfort and the vehicle stability. 


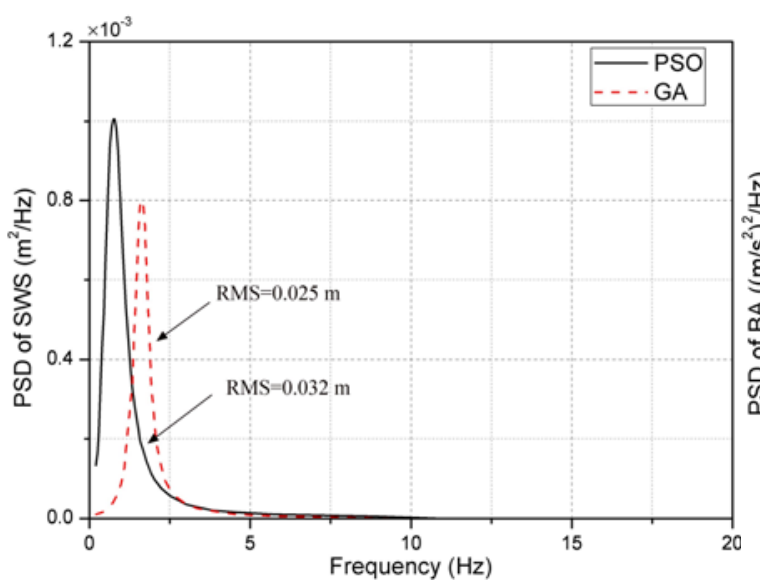

(a) SWS

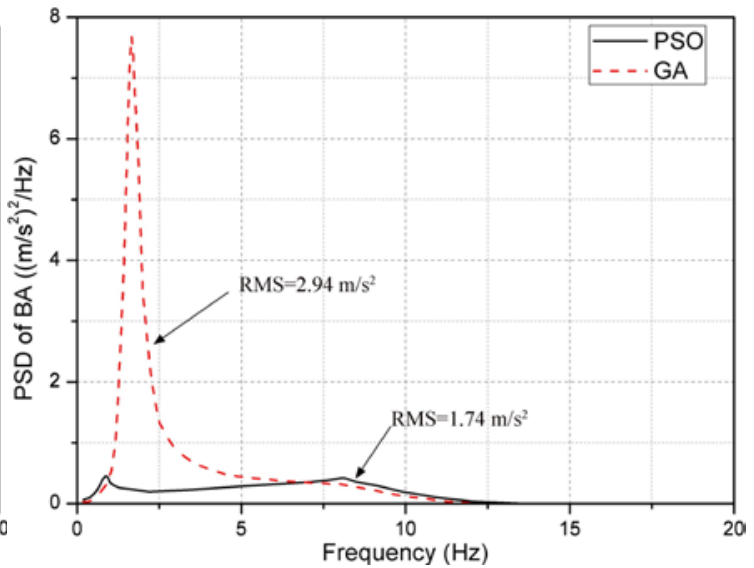

(b) BA

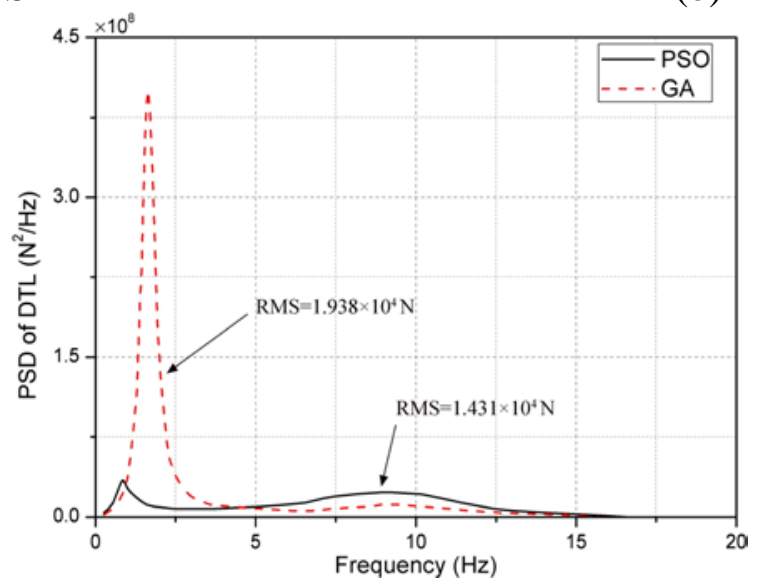

(c) DTL

Fig. 5 Power spectral density function response under road random excitation

\section{Summary}

From road friendliness perspective, the Particle Swarm Optimization technique is proposed for the enhancement of the passive suspension performance of heavy vehicles. Minimizing the dynamic tyre load is used as the objective of optimization problem instead of ride quality, and three design parameters including the tyre stiffness, the suspension stiffness and the damping coefficient are considered. The optimum design parameters for heavy vehicle suspension are obtained by PSO to be: $k_{\mathrm{s}}=123,807 \mathrm{~N} / \mathrm{m}, k_{t}=1,997,216 \mathrm{~N} / \mathrm{m}$ and $c_{\mathrm{s}}=21,053 \mathrm{~N} . \mathrm{s} / \mathrm{m}$. Suspension performance criteria such as SWS, BA and DTL are estimated under bump and random road profile disturbances. The results show that the suspension obtained using PSO can waste the energy due to bump excitation effectively, cut down the settling time, and improve both the ride comfort and vehicle stability compared with the suspension obtained using GA. In fact, there will be more than three design parameters for a more realistic model. GA will be difficult to find the optimum parameters with small scale population. In this situation, PSO will still be an effective approach.

\section{References}

[1]. R. Rajamani, Vehicle Dynamics and Control, Springer Science and Business Media, New York, (2006).

[2]. A.N. Heath, M.G. Good, Heavy vehicle design parameters and dynamic pavement loading, Australian Road Research. 15 (1985) 249-263.

[3]. T.D. Gillespie, Fundamentals of Vehicle Dynamics, Society of Automotive Engineers, Warrendale, (1992).

[4]. W.W. Chooi, S.O. Oyadiji, Mathematical modelling, analysis and design of magnetorheological (MR) dampers, ASME J. Vib. Acoust. 131 (2009) 061002-10. 
[5]. H.S. Lee, and B.S. Choi, Control and response characteristics of a magnetorheological fluid damper for passenger vehicles, J. Intel. Mat. Syst. Str. 11 (2000) 80-87.

[6]. D. Simon, M. Ahmadian, Vehicle evaluation of the performance of magnetorheological dampers for heavy truck suspensions, ASME J. Vib. Acoust. 123 (2001) 365-375.

[7]. T.D. Gillespie, Effects of heavy vehicle characteristics on pavement response and performance, National Corporative Highway Research Program, Report 353, (1993).

[8]. D. Cebon, Interaction between heavy vehicles and roads, Society of Automotive Engineers, SAE Technical Paper, No. 93001. (1993).

[9]. L. Sun, X. Deng, Predicting vertical dynamic loads caused by vehicle-pavement interaction, American Society of Civil Engineers, J. Transp. Eng. 124 (1998) 470-478.

[10]. J.H.F. Woodrooffe, P.A. Leblanc, The influence of suspension variations on dynamic wheel loads of heavy vehicles, SAE Technical Paper, No. 861973. (1986).

[11]. L. Sun, X. Cai, J. Yang, Genetic algorithm-based optimum vehicle suspension design using minimum dynamic pavement load as a design criterion, J. Sound. Vib. 301 (2007) 18-27.

[12]. J.K. Paeng, J.S. Arora, Dynamic response optimization of mechanical systems with multiplier methods, American Society of Mechanical Engineers, J. Mech. Trans. Auto. Des. 111 (1989) 73-80.

[13]. W.L. Winston, Operations Research: Applications and Algorithms, third ed., Duxbury Press, Belmont, (1994).

[14]. A.E. Baumal, J.J. McPhee, Application of genetic algorithms to the design optimization of an active vehicle suspension system, Comput. Method. Appl. Mech. Eng. 163 (1998) 87-94.

[15]. O. Gundogdu, Optimal seat and suspension design for a quarter car with driver model using genetic algorithms, Int. J. Ind. Ergonom. 37 (2007) 327-332.

[16]. M. Zehsaz, M.H. Sadeghi, M.M. Ettefagh, Tractor cabin's passive suspension parameters optimization via experimental and numerical methods, J. Terramechanics. 48 (2011) 439-450.

[17]. J. Kennedy, R.C. Eberhart, Particle swarm optimization, Proceedings of IEEE International Conference on Neural Networks, 4, 1942-1948, Perth, Austrailia, December. (1995)

[18]. J. Kennedy, The particle swarm: social adaptation of knowledge, Proceedings of IEEE International Conference on Evolutionary Computation, Indianapolis, IN, April. (1997)

[19]. M. Sunwoo, K.C. Cheok, N.J. Huang, Model reference adaptive control for vehicle active suspension systems, Institute of Electrical and Electronics Engineers Transactions on Industrial Electronics. 38 (1991) 217-222.

[20]. Y. Shi, R.C. Eberhart, Parameter selection in particle swarm optimization, Evolutionary Programming VII, Lecture Notes in Computer Science, Springer, 1447 (1998) 591-600.

[21]. I.C. Trelea, The particle swarm optimization algorithm: convergence analysis and parameter selection, Information Processing Letters. 85 (2003) 317-25.

[22]. S.B. Choi, W.Q. Zhu, W.K. Kim, Vibration control of a semi-active suspension featuring electrorheological fluid dampers, J. Sound. Vib. 234 (2000) 537-546.

[23]. M.M. ElMadany, An analytical investigation of isolation systems for cab ride, Comput. Struct. 27 (1987) 679-688. 